\title{
Isolation of dendritic cells from umbilical cord blood using magnetic activated cell sorting or adherence
}

\author{
YACHUN BIE, QIUXIANG XU and ZHENYU ZHANG
}

Department of Obstetrics and Gynecology, Beijng Chao-Yang Hospital, Capital Medical University, Beijing 100020, P.R. China

Received June 30, 2014; Accepted March 17, 2015

DOI: $10.3892 / \mathrm{ol} .2015 .3198$

\begin{abstract}
Dendritic cells (DCs) are a highly specialized type of antigen-presenting cell. The present study describes and compares two methods for preparing DCs from umbilical cord blood. The first method involves the isolation of DCs by magnetic activated cell sorting (MACS). This technique isolates $\mathrm{CD}_{3} 4^{+}$cells from cord blood and induces the formation of DCs by the addition of cytokines, granulocyte macrophage colony-stimulating factor and interleukin-4. The second method involves the generation of large numbers of DCs from cord blood using an adherent method, which isolates umbilical cord blood mononuclear cells and induces DCs in the same conditions as those used in MACS. The DCs were harvested following 7 days of incubation and observed with an inverted microscope. The phenotype of the cells was then analyzed by flow cytometry. The results revealed that, subsequent to 7 days of incubation, the differentiated DCs obtained using the adherent method were more mature than those isolated using MACS. However, these cells were unable to be maintained in culture for more than 9-10 days. By contrast, the DCs derived from $\mathrm{CD} 34^{+}$cells by MACS were phenotypically stable and could be maintained for up to 3 weeks in culture. Either method produced DCs from cord blood. However, the DCs isolated using the MACS method demonstrated higher homogeneity, yield and viability than those obtained using the adherent method. Due to the various compositions of the monocyte subsets isolated, isolation methods affect the phenotypes and functions of the resultant DCs.
\end{abstract}

\section{Introduction}

Dendritic cells (DCs) were first identified in the early 1970s (1). Since then, it has been established that DCs are the primary antigen-presenting cells (APCs) to T-lymphocytes,

Correspondence to: Dr Zhenyu Zhang, Department of Obstetrics and Gynecology, Beijng Chao-Yang Hospital, Capital Medical University, 8 Gongti South Road, Beijing 100020, P.R. China E-mail: bjzhangzhy@sina.cn

Key words: dendritic cells, umbilical cord blood mononuclear cells, magnetic activated cell sorting, adherence which initiate and also regulate cellular immune responses (2). Immunotherapy using DCs has been investigated in a number of cancers during the last two decades. The results of these studies have revealed the induction of tumor-specific immune responses. DCs can be isolated ex vivo from blood mononuclear cells using granulocyte macrophage colony-stimulating factor (GM-CSF) and interleukin-4 (IL-4) (3). However, DCs are usually present in extremely small numbers in the circulation, and exhibit marked diversity. There are a number of previous studies that have described methods for culturing DCs in vitro (4-6). The cells can be generated from various cellular sources, including bone marrow, umbilical cord blood and peripheral blood. CD $34^{+}$hematopoietic progenitor cells are often used as an alternative source of DCs, cord blood is rich in these cells. Although the cellular sources and culture conditions are diverse, the majority of protocols generate DCs using GM-CSF, IL-4 and tumor necrosis factor (TNF)- $\alpha(7,8)$.

The present study compares two different mononuclear cell isolation methods to obtain DCs from cord blood, namely plastic adherence and magnetic activated cell sorting (MACS). The results revealed that either method is able to produce DCs, but that the cells differ in their differentiation pathways, phenotypes and functions. The differentiated DCs obtained by the adherent method were more mature than those isolated by MACS.

\section{Materials and methods}

Cord blood collection. Subsequent to obtaining written informed consent, $50 \mathrm{ml}$ samples of human umbilical cord blood were collected from healthy, full-term deliveries. All mothers and infants were healthy and demonstrated no abnormal laboratory results. This study was approved by the ethics committee of Beijing Chao-Yang Hospital, Capital Medical University (Beijing, China).

Mononuclear cell isolation. The umbilical cord blood mononuclear cells (UBMCs) were isolated from fresh cord blood with $200 \mathrm{IU} / \mathrm{ml}$ heparin using $1.077 \mathrm{~g} / \mathrm{ml}$ Ficoll-Hypaque (Gibco-Invitrogen, Paisley, UK) density gradient centrifugation, and then centrifuged for $30 \mathrm{~min}$ at $700 \mathrm{x} \mathrm{g}$ at room temperature.

$M A C S$. The UBMCs were isolated by the positive selection of $\mathrm{CD}_{3} 4^{+}$cells using an immunomagnetic separation kit 
(MiniMACS CD34 Isolation kit; Miltenyi Biotec GmbH, Bergisch Gladbach, Germany), according to the manufacturer's instructions. The cells isolated by the MACS method were washed twice with phosphate-buffered saline (PBS; GE Healthcare Life Sciences, Logan, UT, USA) and then seeded into six-well culture plates (Costar, Cambridge, MA, USA) at a density of $1 \times 10^{6} / 2 \mathrm{ml}$ per well. The cells were cultured in RPMI-1640 medium containing 10\% fetal bovine serum (FBS; Gibco Life Technologies, Carlsbad, CA, USA) and supplemented with $1000 \mathrm{U} / \mathrm{ml}$ recombinant human GM-CSF (PeproTech, Inc., Rocky Hill, NJ, USA) and 1000 U/ml IL-4 (PeproTech, Inc.) at $37^{\circ} \mathrm{C}$ in an incubator with a humidified $5 \% \mathrm{CO}_{2}$ atmosphere.

Adherence method. The UBMCs were seeded into six-well culture plates containing RPMI-1640 medium and 10\% FBS at a density of $1 \times 10^{6} / 2 \mathrm{ml}$ per well. After $2 \mathrm{~h}$ incubation at $37^{\circ} \mathrm{C}$ in a humidified incubator with a $5 \% \mathrm{CO}_{2}$ atmosphere, the non-adherent cells were removed. The adherent cells were then further cultured in RPMI-1640 medium containing 10\% FBS, $1000 \mathrm{U} / \mathrm{ml}$ recombinant human GM-CSF and $1000 \mathrm{U} / \mathrm{ml} \mathrm{IL}-4$ at $37^{\circ} \mathrm{C}$ in an incubator with a humidified $5 \% \mathrm{CO}_{2}$ atmosphere.

Generation of dendritic cells. Every three days, $50 \%$ of the spent medium was replaced with fresh medium containing GM-CSF and IL-4 in order to yield final concentrations of $1000 \mathrm{U} / \mathrm{ml}$. On the 5th day, the cells were cultured in TNF- $\alpha$ (PeproTech, Inc.) for a further two days. Following 7 days of culture, the DCs were harvested.

Flow-based analysis of labeled cells. The DCs were washed twice with PBS and then incubated in a $10 \% \mathrm{Fc}$ receptor $(\mathrm{FcR})$ blocking solution (Miltenyi Biotec $\mathrm{GmbH}$ ) for $30 \mathrm{~min}$ at $4^{\circ} \mathrm{C}$ in order to block non-specific binding to the FcR. The cells were then stained with phycoerythrin (PE)-conjugated monoclonal mouse anti-human-cluster of differentiation (CD)80 (1:100; cat. no. ab-155374; Abcam, Cambridge, MA, USA) and monoclonal mouse anti-human human leukocyte antigen (HLA)-DR (1:100; cat. no. ab95830; Abcam) antibodies or APC-conjugated monoclonal mouse anti-human CD11c antibodies (1:100; cat. no. 657713; BD Biosciences, Franklin Lakes, NJ, USA), whilst control staining was performed using isotype-matched, irrelevant antibodies that were also directly conjugated to PE or APC. The fluorescence intensity of the cells was analyzed using a FACS Calibur flow cytometry system (BD Biosciences).

Statistical analysis. The data in each figure corresponds to one representative experiment of at least three independent experiments. A $t$-test was used to determine the significance of data comparison. A one-way analysis of variance was used for the statistical analysis of differences among the experimental groups. All statistical analyses were performed using SPSS 19.0 statistical software (SPSS, Inc., Chicago, IL, USA). A value of $\mathrm{P}<0.05$ was used to indicate a statistically significant difference.

\section{Results}

Following 7 days of incubation, microscopic images of the DCs were captured with an inverted microscope (IX51; Olympus Corporation, Tokyo, Japan) using the $\mathrm{x} 10$ or $\mathrm{x} 20$ objective (Figs. 1-4).

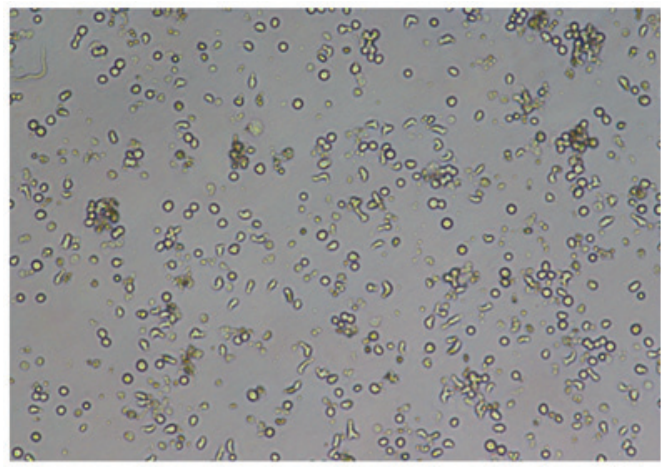

Figure 1. Dendritic cells isolated by magnetic activated cell sorting on the 7 th day of culture (magnification, $\mathrm{x} 10$ ).

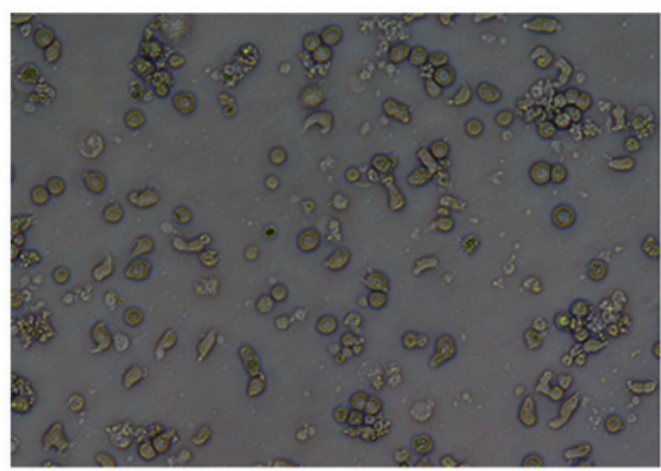

Figure 2. Dendritic cells isolated by magnetic activated cell sorting on the 7 th day of culture (magnification, $\mathrm{x} 20$ ).

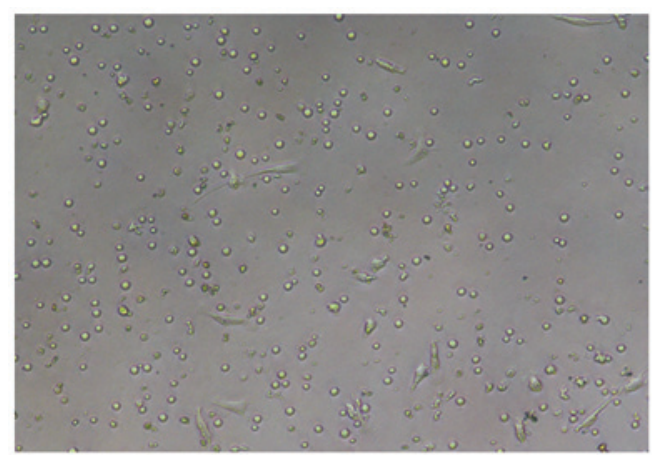

Figure 3. Dendritic cells isolated by adherence on the 7th day of culture (magnification, x10).

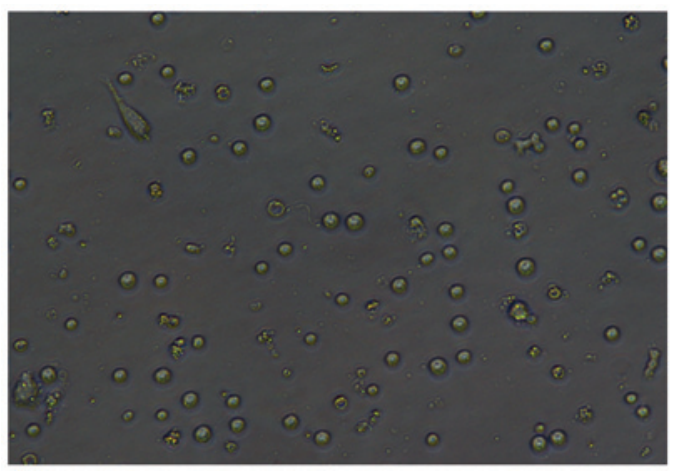

Figure 4. Dendritic cells isolated by adherence on the 7th day of culture (magnification, x20). 
A

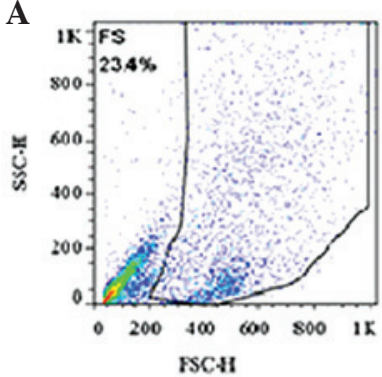

D

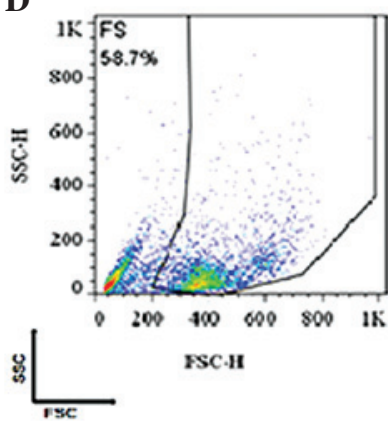

B

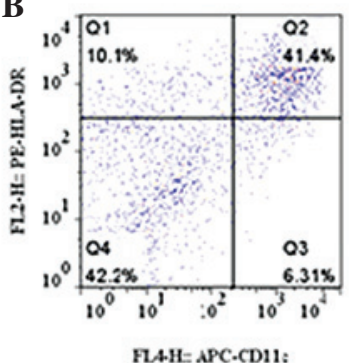

E

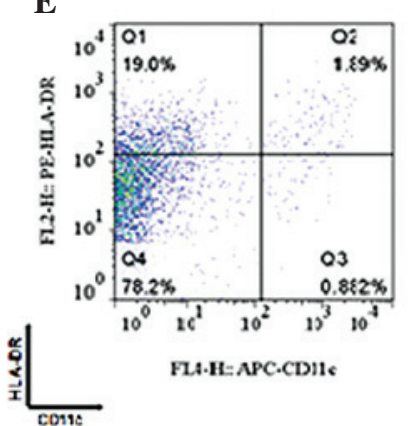

C

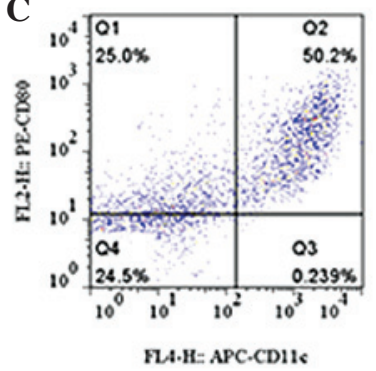

F

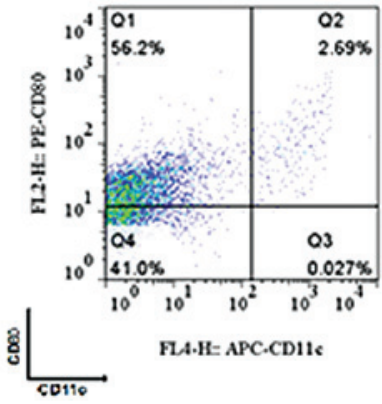

Figure 5. Flow cytometric analysis of the dendritic cells on the 7th day of culture. (A-C) Cells that were cultured by the adherent method and (D-F) cells that were cultured by the magnetic activated cell sorting method. The cells in the gated population were further analyzed and identified to be $(\mathrm{B}$ and $\mathrm{E}) \mathrm{CD} 11 \mathrm{C}^{+} / \mathrm{HLA}-\mathrm{DR}{ }^{+}$

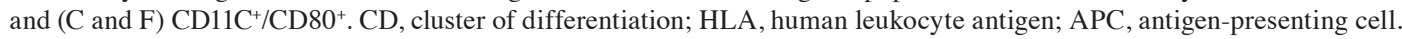

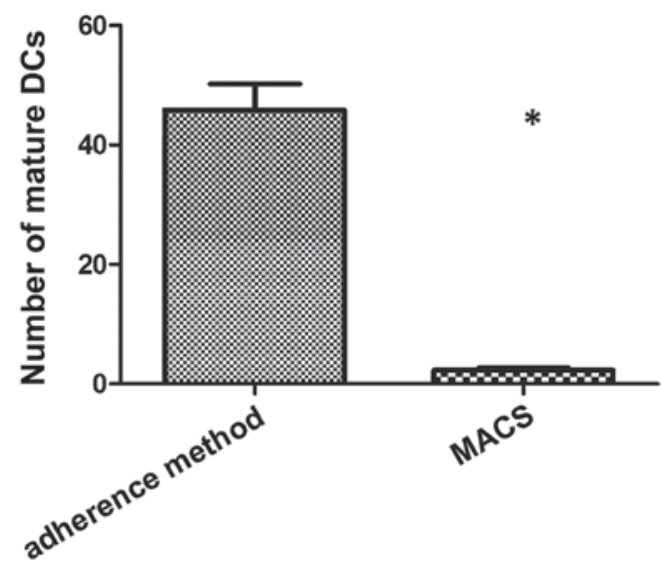

Figure 6. Mature DC ratio under different cultivation methods using cord blood. After 7 days of incubation, the DCs obtained using the adherent method were more mature than those isolated using the MACS method. ${ }^{\mathrm{a}} \mathrm{P}=0.0102$. DC, dendritic cell; MACS, magnetic activated cell sorting.

The results of the flow cytometry analysis are shown in Fig. 5. Fig. 5A-C refer to the cells that were cultured by the adherent method and Fig. 5D-F refers to the cells that were cultured by the MACS method. The cells in the gated population were further analyzed and identified to

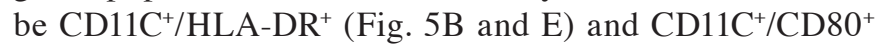
(Fig. 5C and F).

Either method was found to be able to produce DCs. However, subsequent to 7 days of incubation, the DCs differentiated by the adherent method were more mature than those isolated using the MACS method ( $\mathrm{P}=0.0102$; Fig. 6).

In addition, it was identified that the DCs isolated by the adherent method were unable to be maintained in culture for >9-10 days. By contrast, the immature DCs differentiated from
$\mathrm{CD} 4^{+}$cells by MACS were phenotypically stable and could be maintained for up to 3 weeks in culture.

\section{Discussion}

DCs have an important role in the induction of immunity (2). In peripheral tissues, DCs exist as immature cells that must undergo a process of maturation upon exposure to cytokines and antigens, exemplified by the upregulation of major histocompatibility complex (MHC) and costimulatory molecules (CD80/86), activation markers and cytokine production in order to activate $\mathrm{T}$ cells. DCs present acquired antigens to naive $\mathrm{T}$ cells via MHC molecules, and also promote the differentiation and maturation of antibody-producing B cells. DCs exhibit certain common characteristics, including an irregular shape, a distinct cell-surface phenotype, such as extremely high levels of MHC class II proteins, active motility, and potent stimulatory activity for T cell-dependent responses (9). However, they also demonstrate marked heterogeneity (10). The subsets of DCs include cells in lymphoid organs, blood DCs, Langerhans cells, and cells in non-lymphoid organs, such as the lung, gut, heart and synovium (11). In total, two types of DCs have been identified in the blood, namely myeloid DCs (CD11C $\left.{ }^{+} / \mathrm{CD}^{-} 3^{-}\right)$

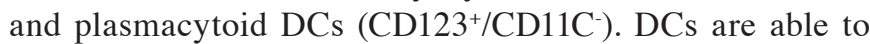
differentiate from a diverse range of progenitors and precursors under appropriate culture conditions $(11,12)$.

Preliminary studies concerning DCs have been hampered by the limited number of cells that are able to be purified $(13,14)$. In practice, DCs can be generated from two primary cell types: CD $34^{+}$cells and monocytes (13). There are a number of ways to isolate monocytes, including plastic adherence and specific marker-based separation techniques, such as MACS and fluorescent activated cell sorting (15). However, DCs derived 
from $\mathrm{CD}_{3} 4^{+}$cells or monocytes differ in their differentiation pathways, phenotype and functions (16-18). A study by Delirezh et al (19) compared the phagocytic activity of two types of cells. The results revealed that the phagocytic activity of DCs obtained by the MACS method was higher than that of the cells isolated by the adherent method. This may be explained by differences in the maturity of the two types of DCs.

It has been established that populations of human monocytes can be divided into two different subsets, namely CD14- ${ }^{-}$D $16^{+}(5-10 \%)$ and CD14+ ${ }^{+}$CD16- $(90-95 \%)(20,21)$. These two types of monocytes differ in their phagocytic activity. The majority of previous studies have used the MACS method to separate CD $34^{+}$cells from cord blood in order to induce DCs $(13,16-18,22)$. Others have used an adherent method to induce the differentiation of DCs from peripheral blood or bone marrow $(23,24)$. The present study compared the two methods using cord blood and concluded that the adherent method is a simple technique that is able to induce the formation of DCs. Despite this, the DCs derived from $\mathrm{CD}_{3} 4^{+}$cells by MACS were phenotypically stable and could be maintained for a longer period of time in culture; a feature suitable for research purposes. In terms of clinical applications, the adherence method may be preferable, due to less manipulation and the absence of exposure to the magnetic field of the MACS apparatus (19).

DCs isolated by the MACS method demonstrated higher homogeneity. Furthermore, the yield and viability were markedly increased compared with the DCs isolated using the adherent methods. Differentiating DCs using MACS is, however, more technically demanding than adherence. Monocyte isolation methods affect the phenotypes and functions of resultant DCs due to the different compositions of the monocyte subsets that are isolated. Additional studies should be conducted in order to establish a clear understanding of DCs. Isolation methods should be selected according to the specific clinical or experimental purpose.

\section{References}

1. Steinman RM and Cohn ZA: Identification of a novel cell type in peripheral lymphoid organs of mice. I. Morphology, quantitation, tissue distribution. J Exp Med 137: 1142-1162, 1973.

2. Banchereau J, Briere F, Caux C, et al: Immunobiology of dendritic cells. Annu Rev Immunol 18: 767-811, 2000.

3. Schuler G, Schuler-Thurner B and Steinman RM: The use of dendritic cells in cancer immunotherapy. Curr Opin Immunol 15: 138-147, 2003.

4. Chu CS, Boyer J, Schullery DS, et al: Phase I/II randomized trial of dendritic cell vaccination with or without cyclophosphamide for consolidation therapy of advanced ovarian cancer in first or second remission. Cancer Immunol Immunother 61: 629-641, 2012.

5. Coosemans A, Vanderstraeten A, Tuyaerts S, et al: Wilms' Tumor Gene 1 (WT1) - loaded dendritic cell immunotherapy in patients with uterine tumors: a phase I/II clinical trial. Anticancer Res 33: 5495-5500, 2013

6. Mody N, Dubey S, Sharma R, Agrawal U and Vyas SP: Dendritic cell-based vaccine research against cancer. Expert Rev Clin Immunol 11: 213-232, 2015.
7. GanjiBakhsh M, Nejati V, Delirezh N, Asadi M and Gholami K: Mixture of fibroblast, epithelial and endothelial cells conditioned media induce monocyte-derived dendritic cell maturation. Cell Immunol 272: 18-24, 2011.

8. Delirezh N, Majedi L, Asri Rezaei S and Ranjkeshzadeh H: Generation of mature monocyte-derived dendritic cells in the presence of heparin and monocyte conditioned medium: phenotypic and functional comparison. Iran Biomed J 15: 79-84, 2011.

9. Takamizawa M, Rivas A, Fagnoni F, et al: Dendritic cells that process and present nominal antigens to naive $\mathrm{T}$ lymphocytes are derived from CD2 $2^{+}$precursors. J Immunol 158: 2134-2142, 1997.

10. Ito T, Inaba M, Inaba K, et al: A CD1a+/CD11c+ subset of human blood dendritic cells is a direct precursor of Langerhans cells. J Immunol 163: 1409-1419, 1999.

11. Cella M, Jarrossay D, Facchetti F, et al: Plasmacytoid monocytes migrate to inflamed lymph nodes and produce large amounts of type I interferon. Nat Med 5: 919-923, 1999.

12. Oehler L, Berer A, Keil F, et al: Generation of dendritic cells from human chronic myelomonocytic leukemia cells in fetal calf serum-free medium. Leuk Lymphoma 38: 577-586, 2000.

13. Ferlazzo G, Wesa A, Wei WZ and Galy A: Dendritic cells generated either from $\mathrm{CD} 34^{+}$progenitor cells or from monocytes differ in their ability to activate antigen-specific $\mathrm{CD} 8^{+} \mathrm{T}$ cells. $\mathrm{J}$ Immunol 163: 3597-3604, 1999.

14. Broxmeyer HE, Hangoc G, Cooper S, et al: Growth characteristics and expansion of human umbilical cord blood and estimation of its potential for transplantation in adults. Proc Natl Acad Sci USA 89: 4109-4113, 1992.

15. Van Brussel I, Ammi R, Rombouts M, et al: Fluorescent activated cell sorting: An effective approach to study dendritic cell subsets in human atherosclerotic plaques. J Immunol Methods 417: 76-85, 2015.

16. Caux C, Massacrier C, Vanbervliet B, et al: CD34+ hematopoietic progenitors from human cord blood differentiate along two independent dendritic cell pathways in response to granulocyte-macrophage colony-stimulating factor plus tumor necrosis factor alpha: II. Functional analysis. Blood 90: 1458-1470, 1997.

17. Canque B, Camus S, Dalloul A, et al: Characterization of dendritic cell differentiation pathways from cord blood CD34(+) CD7(+)CD45RA(+) hematopoietic progenitor cells. Blood 96: 3748-3756, 2000.

18. Ferlazzo G, Klein J, Paliard X, Wei WZ and Galy A: Dendritic cells generated from CD34+ progenitor cells with flt3 ligand, c-kit ligand, GM-CSF, IL-4 and TNF-alpha are functional antigen-presenting cells resembling mature monocyte-derived dendritic cells. J Immunother 23: 48-58, 2000.

19. Delirezh N, Shojaeefar E, Parvin P and Asadi B: Comparison the effects of two monocyte isolation methods, plastic adherence and magnetic activated cell sorting methods, on phagocytic activity of generated dendritic cells. Cell J 15: 218-223, 2013.

20. Delirezh N and Shojaeefar E: Phenotypic and functional comparison between flask adherent and magnetic activated cell sorted monocytes derived dendritic cells. Iran J Immunol 9: 98-108, 2012.

21. Mucci I, Legitimo A, Compagnino M, et al: The methodological approach for the generation of human dendritic cells from monocytes affects the maturation state of the resultant dendritic cells. Biologicals 37: 288-296, 2009.

22. Ackermann B, Tröger A, Glouchkova L, Körholz D, Göbel U and Dilloo D: Characterization of $\mathrm{CD} 34^{+}$progenitor-derived dendritic cells pulsed with tumor cell lysate for a vaccination strategy in children with malignant solid tumors and a poor prognosis. Klin Padiatr 216: 176-182, 2004.

23. Schott M, Seissler J, Feldkamp J, von Schilling C and Scherbaum WA: Dendritic cell immunotherapy induces antitumour response in parathyroid carcinoma and neuroendocrine pancreas carcinoma. Horm Metab Res 31: 662-664, 1999.

24. DeMatos P, Abdel-Wahab Z, Vervaert C, Hester D and Seigler H: Pulsing of dendritic cells with cell lysates from either B16 melanoma or MCA-106 fibrosarcoma yields equally effective vaccines against B16 tumors in mice. J Surg Oncol 68: 79-91, 1998. 